\title{
Mitochondrial myopathy with reversible cytochrome C oxidase deficiency
}

INSERM

\section{Source}

INSERM. (1999). Orphanet: an online rare disease and orphan drug data base.

Mitochondrial myopathy with reversible cytochrome C oxidase deficiency.

ORPHA:254864

A rare, genetic, mitochondrial oxidative phosphorylation disorder characterized by a potentially life-threatening, severe myopathy manifesting in the neonatal to early infantile period, followed by marked, spontaneous improvement of muscular function by early childhood. Associated biochemical findings include lactic acidosis and a transient, marked decrease in respiratory chain activity. 